\title{
Presence of accessory abductor digiti minimi muscle in two cadavers
}

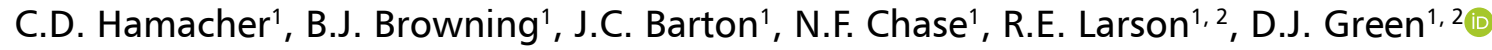 \\ ${ }^{1}$ Campbell University School of Osteopathic Medicine, Buies Creek, United States \\ ${ }^{2}$ Department of Anatomy, Buies Creek, United States
}

[Received: 25 October 2021; Accepted: 23 December 2021; Early publication date: 17 January 2022]

\begin{abstract}
During routine cadaveric dissection, accessory hypothenar muscles were incidentally discovered in two cadavers, both males, aged 86 and 92. Both muscles originated from the palmaris longus tendon in the distal portion of the forearm and were identified as accessory abductor digiti minimi (AADM) muscles, based on their association with abductor digiti minimi. While AADM is a common variant in the antebrachium, it is less typical for them to originate from the palmaris longus tendon. The presence of such an AADM could complicate surgical procedures requiring resection of the palmaris longus tendon. Moreover, the surrounding neurovasculature - namely the ulnar nerve as it passes through the ulnar canal between the pisiform and hook of the hamate - could be compressed by contractions of an AADM with such a proximal origin. This can manifest as ulnar neuropathies resulting in pain, weakness, or protracted flexion of the fourth and fifth digits (ulnar claw). Our description of these muscles adds to previous accounts of variation of the palmaris longus and abductor digiti minimi muscles while considering potential clinical implications. (Folia Morphol 2023; 82, 1: 216-220)
\end{abstract}

Key words: anatomy, dissection, variation, forearm, wrist, hand, palm, medicine

\section{INTRODUCTION}

The hypothenar muscles of the hand include abductor digiti minimi, flexor digiti minimi, and opponens digiti minimi. All are innervated by the ulnar nerve, take origin from the medial carpus or flexor retinaculum, and serve to abduct, flex, and oppose the fifth digit, respectively (Table 1). Here, we report on two separate cases of accessory abductor digiti minimi (AADM) muscles discovered incidentally during routine cadaveric dissection. Previous discussion of AADM describe the muscle as originating from the flexor retinaculum, pisiform, flexor carpi radialis, or antebrachial fascia, with the latter being the most common origin site [5]. In contrast, both AADM muscles described in this report originated from the palmaris longus tendon. Palmaris longus is a slender muscle located in the superficial portion of the anterior antebrachium. It shares a common muscle belly of origin - from the medial epicondyle of the humerus - with other superficial flexors of the elbow and wrist: pronator teres, flexor carpi radialis, flexor carpi ulnaris, and flexor digitorum superficialis; its insertion tendon joins the palmar aponeurosis after passing superficial to the flexor retinaculum. The AADM muscles described in this report followed a more extensive course than other such variants [12] and given their more proximal origin site relative to the other hypothenar muscles, passed superficially to

Address for correspondence: Mr. B.J. Browning and Dr. D.J. Green, Campbell University School of Osteopathic Medicine, PO Box 4280 27506 Buies Creek, United States, e-mail: b_browning0620@email.campbell.edu and dgreen@campbell.edu

This article is available in open access under Creative Common Attribution-Non-Commercial-No Derivatives 4.0 International (CC BY-NC-ND 4.0) license, allowing to download articles and share them with others as long as they credit the authors and the publisher, but without permission to change them in any way or use them commercially. 
Table 1. Origins, insertions, and actions of hypothenar muscles and the accessory abductor digiti minimi (AADM) variant discussed in this report, which are all innervated by the ulnar nerve

\begin{tabular}{|c|c|c|c|}
\hline Muscle & Origin & Insertion & Action \\
\hline Abductor digiti minimi & Pisiform; flexor carpi ulnaris tendon & $\begin{array}{l}\text { Medial aspect of base } \\
\text { of } 5^{\text {th }} \text { proximal phalanx }\end{array}$ & Abduction of the $5^{\text {th }}$ digit \\
\hline AADM & $\begin{array}{l}\text { Variable: palmaris longus tendon*; flexor retinaculum; } \\
\text { pisiform; flexor carpi radialis; antebrachial fascia }\end{array}$ & Abductor digiti minimi muscle belly & Abduction of the $5^{\text {th }}$ digit \\
\hline Flexor digiti minimi & Hook of hamate; flexor retinaculum & $\begin{array}{l}\text { Medial aspect of base of } 5^{\text {th }} \text { proximal } \\
\text { phalanx }\end{array}$ & Flexion of the $5^{\text {th }}$ digit \\
\hline Opponens digiti minimi & Hook of hamate; flexor retinaculum & Medial aspect of $5^{\text {th }}$ metacarpal & $\begin{array}{l}\text { Lateral rotation and opposition } \\
\text { of } 5^{\text {th }} \text { digit }\end{array}$ \\
\hline
\end{tabular}

*Indicates the origin of the AADMs described in this report.

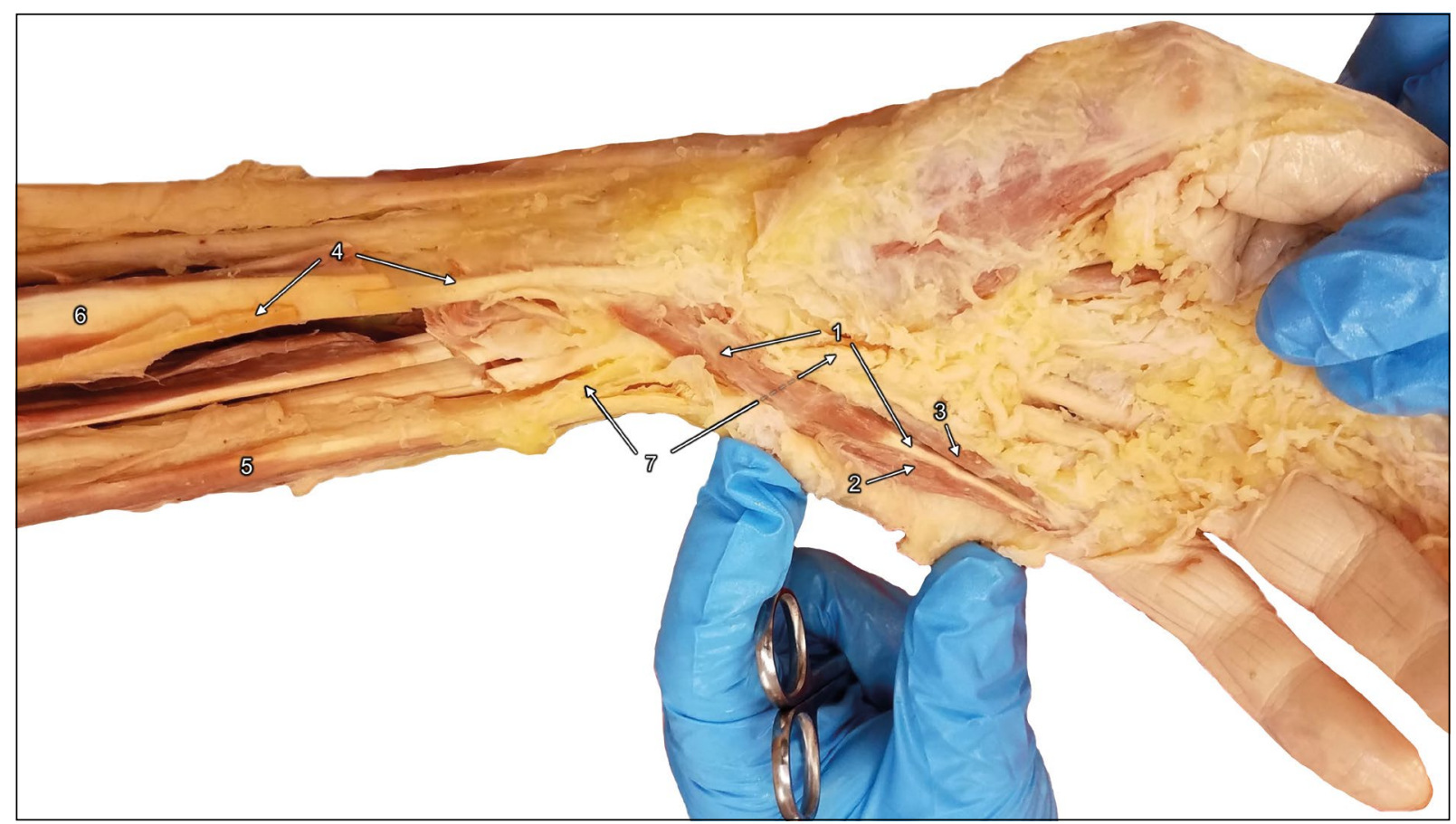

Figure 1. Distal forearm, wrist, and hand of a 92-year-old male cadaver. Note the presence of the accessory abductor digiti minimi (label no. 1) originating from the palmaris longus tendon (no. 4), which crosses medially towards digit five while passing superficial to the ulnar artery and nerve (no. 7). Labels: 1 - accessory abductor digiti minimi; 2 - abductor digiti minimi; 3 - flexor digiti minimi; 4 — palmaris longus (sectioned); 5 - flexor carpi ulnaris; 6 - flexor carpi radialis; 7 - ulnar artery and nerve.

the ulnar nerve and artery. In reviewing the antebrachial anatomy presented in these two cadavers, we consider the potential clinical implications of these muscle variants.

\section{CASE REPORT}

During routine anatomical dissection, nearly identical, anomalous forearm musculature was discovered bilaterally in both 92- and 86-year-old male cadaveric specimens (Figs. 1, 2, respectively). These muscles both originated from the palmaris longus tendons in the anterior forearm and passed superficially to the ulnar canal to insert on the fifth proximal phalanx.
Based largely on their proximity to abductor digiti minimi and pattern of insertion, we determined that the anomalous muscles are best classified as AADM $[3-5,9,11,12,14]$. AADM is the most common variant of the hypothenar muscles; however, having this variant arise from the palmaris longus tendon is somewhat rare [5]. More typically, the AADM arises from either the flexor retinaculum, pisiform, flexor carpi radialis, or the antebrachial fascia, which is the most common origin site [5]. As with the rest of the hypothenar musculature, the neurovascular supply is distributed through branches of the ulnar nerve and artery [11]. The majority of AADM variants are thin, 


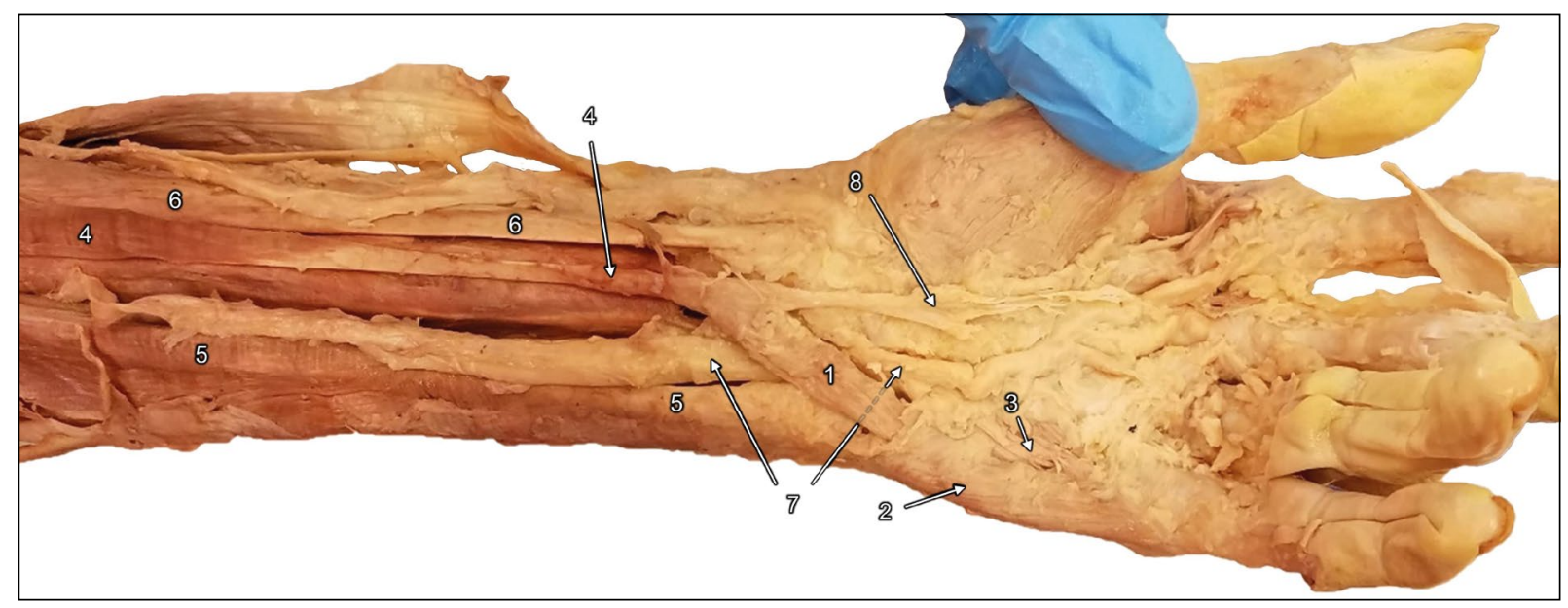

Figure 2. Distal forearm, wrist, and hand of an 86 -year-old male cadaver. Just as in Figure 1, note how the accessory abductor digiti minimi (label no. 1) originates from the palmaris longus tendon (no. 4) and crosses superficial and proximal to where the ulnar artery and nerve (no. 7) enter the ulnar tunnel. Labels: 1 - accessory abductor digiti minimi; 2 - abductor digiti minimi; 3 - flexor digiti minimi; 4 - palmaris longus; 5 - flexor carpi ulnaris; 6 - flexor carpi radialis; 7 - ulnar artery and nerve; 8 - palmar aponeurosis.

with a fascial-like appearance [10]. In contrast, both examples of AADM in the cadavers discussed here presented with distinct muscle fascicles and discernible bellies (Figs. 1, 2). Such a large muscle variant in the superficial forearm could lead to pathological compression of nearby neurovascular tissues. In extreme cases this might require its surgical removal [e.g., 4, 11], yet its typically membranous presentation likely limits the necessity of such interventions in most cases.

\section{DISCUSSION}

This report documents the discovery of two cadavers (out of 27 specimens dissected during the 2019-2020 academic year) presenting with similar versions of the AADM muscle variant during a routine medical school anatomical dissection course. Such a finding implies that these types of muscular variants are not uncommon. Dodds et al. [5] reported a $22.4 \%$ incidence of anomalous muscles associated with the ulnar canal. In fact, these authors note several different presentations of AADM with divergent origin sites: pisiform, antebrachial fascia, palmaris longus tendon, investing fascia of flexor carpi radialis, intermuscular fascia, flexor carpi ulnaris, and flexor retinaculum. Other noted variants include fusion with flexor digiti minimi, and two or more heads of origin, each of which may arise from any of the anatomical structures listed above [11].

Both AADM muscle variants described here originated from the palmaris longus tendon. The modest size of palmaris longus provides limited contri- butions to elbow and wrist flexion; however, given its absence in approximately $11.7 \%$ of forearms, its functional role may be redundant; consequently, it is commonly harvested for use as a tendon graft, and in the treatment of mallet finger, ptosis, and other reconstructive surgeries $[2,7,8,13]$. While it is unclear how functionally active the AADMs identified in these two cadavers would have been relative to the rest of the hypothenar musculature, their substantial size and visible muscle striations suggest they had a reasonably significant role. Furthermore, had either of these individuals required surgical harvest of the palmaris longus tendon, they may have experienced weakness or other undesirable effects on hand function resulting from potential loss of AADM. Given the prevalence of this variant and the potential for its origin from the antebrachial fascia or palmaris longus, diagnostic imaging using ultrasound or magnetic resonance imaging (MRI) would be critical to reduce the risk of complications prior to the surgical resection of palmaris longus.

In contrast to the potential functional role of AADM, variability of musculature in the volar wrist could lead to neuropathy if the ulnar nerve is compressed along its course. The ulnar canal, formed by the volar carpal ligament, transverse carpal ligament, pisiform, and the hook of the hamate, provides a relatively small passageway for the ulnar nerve and associated vasculature to reach the hand [1]. Patients with ulnar canal syndrome typically present with a combination of sensory symptoms and/or motor symptoms of the fourth and fifth digits, in- 
cluding burning, tingling, weakness, or numbness [6]. The role of muscle variants like AADM should be a consideration in patients presenting with otherwise indeterminate neuropathy of the wrist or forearm [9]. There are many well-documented reports of accessory muscles compressing the ulnar nerve or even the median nerve as it enters the carpal tunnel $[9,11,12]$. These accessory muscles may contribute to nerve damage with symptoms including handgrip weakness, wrist and/or hand pain, or "ulnar claw" [9]. In some instances, resection of the anomalous AADM has been shown to relieve symptoms $[4,5$, $11,12]$. For example, a recent surgical case noted the presence of an AADM ultimately resulting in surgical intervention to treat bilateral carpal and ulnar tunnel syndromes [11]. Imaging modalities like ultrasound or MRI could identify an AADM either incidentally or prior to surgical decompression of an ulnar or median nerve neuropathy $[4,9,11]$. While many AADM muscles are asymptomatic, injury or hypertrophy occurring in individuals with manual labour occupations may increase the risk for neuropathies like ulnar canal syndrome [9]. With pronounced nerve compression, arterial supply is also likely to be compromised, impacting several intrinsic muscles of the hand and potentially leading to "ulnar claw" deformities [7, 12]. Comorbidities like ulnar artery thrombosis or fibrosis, fractures, Dupuytren's contracture, or rheumatoid arthritis may amplify these drastic complications. Detailed medical records (beyond cause of death) were unavailable to us, however, so we cannot assess if these AADM muscles presented the donors with any of the musculoskeletal issues listed above.

\section{CONCLUSIONS}

During routine cadaveric dissection, the presence of two AADM muscles was identified in two separate individuals. While the discovery of these muscles is not unusual in and of itself, the fact that they both originated from palmaris longus is noteworthy [5]. AADM variants are common, and in the cases we described above, may complicate wrist or hand surgeries for compression neuropathy or when surgical harvesting of the palmaris longus tendon is required. Diagnostic imaging like ultrasound or MRI may limit the risk of such complications, but AADMs are often thin and fascial (unlike the two variants described above) and may be hard to discern using lower resolution imaging modalities.
The ulnar nerve and artery are most commonly compressed by location of typical AADM variants, though the median nerve is also known to be affected, albeit less extensively [5]. To this end, in patients presenting with clinical evidence of ulnar neuropathy, such as medial forearm or hand neuralgia, hand grip weakness, "ulnar claw" syndrome or other indeterminate hand or forearm symptoms, ultrasound or MRI may be used to determine if a muscle variant like AADM may be exacerbating the condition. In cases where an AADM variant becomes symptomatic, surgical resection has been shown to provide long term resolution.

\section{Acknowledgements}

We wish to thank these two donors and their families, in particular, and all of the donors who contributed to our anatomical education. We are grateful for the assistance of Dr. Bonnie Brenseke and Mrs. Ashley Valley. We appreciate the encouragement of our editor, Prof. Janusz Moryś, and an anonymous Reviewer. Finally, we wish to acknowledge the Faculty and Administration of the Campbell University School of Osteopathic Medicine for their support of this project.

\section{Conflict of interest: None declared}

\section{REFERENCES}

1. Aleksenko D, Varacallo M. 2021 [Updated Jul 18]. Guyon Canal Syndrome. In: StatPearls [Internet]. Treasure Island (FL): StatPearls Publishing. https://www.ncbi.nlm.nih.gov/ books/NBK431063/.

2. Berger $P$, Duerinckx J. Flexor pollicis longus tendon rupture after volar wrist plating: reconstruction with palmaris longus interposition graft. Acta Orthop Belg. 2017; 83(3): 467-472, indexed in Pubmed: 30423650.

3. Coraci D, Luchetti R, Paolasso I, et al. Intermittent ulnar nerve compression due to accessory abductor digiti minimi muscle: Crucial diagnostic role of nerve ultrasound. Muscle Nerve. 2015; 52(3): 463-464, doi: 10.1002/mus.24660, indexed in Pubmed: 25808715.

4. Dimitriou C, Natsis K. Accessory abductor digiti minimi muscle causing ulnar nerve entrapment at the Guyon's canal: a case report. Clin Anat. 2007; 20(8): 974-975, doi: 10.1002/ca.20518, indexed in Pubmed: 17583589.

5. Dodds GA, Hale D, Jackson WT. Incidence of anatomic variants in Guyon's canal. J Hand Surg Am. 1990; 15(2): 352-355, doi: 10.1016/0363-5023(90)90122-8, indexed in Pubmed: 2324469.

6. Doherty, T.J. 2021. Ulnar neuropathy at the elbow and wrist. Shefner JM, Goddeau Jr. RP, eds. UpToDate. Waltham, MA: UpToDate Inc. https://www.uptodate. 
com/contents/ulnar-neuropathy-at-the-elbow-and-wrist (Accessed on October 18, 2021).

7. Lam DS, Ng JS, Cheng GP, et al. Autogenous palmaris longus tendon as frontalis suspension material for ptosis correction in children. Am J Ophthalmol. 1998; 126(1): 109-115, doi: 10.1016/s0002-9394(98)00072-5, indexed in Pubmed: 9683156.

8. Liu Z, Ma K, Huang D. Treatment of mallet finger deformity with a modified palmaris longus tendon graft through a bone tunnel. Int J Burns Trauma. 2018; 8(2): 34-39, indexed in Pubmed: 29755840.

9. Lokanathan TH, Surendra M, Ajay N, et al. Unusual co-existence of biaponeurotic palmaris longus and accessory abductor digiti minimi in man. J Clin Diagn Res. 2014; 8(1): 146-147, doi: 10.7860/JCDR/2014/7220.3968, indexed in Pubmed: 24596749.

10. Rixey A, Wenger D, Baffour F, et al. Accessory abductor digiti minimi muscle, less muscular than thought: an update on prevalence, morphology, and review of the literature. Skeletal Radiol. 2021; 50(8): 1687-1695, doi: 10.1007/ s00256-021-03728-5, indexed in Pubmed: 33533963.
11. Slavchev S, Georgiev G. Aberrant abductor digiti minimi muscle found during open surgical decompression of the carpal tunnel: case report. Revista Argentina de Anatomía Clínica. 2016; 5(2): 88-91, doi: 10.31051/1852.8023. v5.n2.14063.

12. Soldado-Carrera F, Vilar-Coromina N, Rodríguez-Baeza A. An accessory belly of the abductor digiti minimi muscle: a case report and embryologic aspects. Surg Radiol Anat. 2000; 22(1): 51-54, doi: 10.1007/s00276-000-0051-1, indexed in Pubmed: 10863748.

13. Yong MW, Yusof N, Rampal L, et al. Prevalence of absence of palmaris longus and its association with gender, hand dominance and absence of FDS tendon to little finger among malay population. J Hand Surg Asian Pac Vol. 2017; 22(4): 484-489, doi: 10.1142/S021881041750054X, indexed in Pubmed: 29117832.

14. Zeiss J, Jakab E, Khimji T, et al. The ulnar tunnel at the wrist (Guyon's canal): normal MR anatomy and variants. AJR Am J Roentgenol. 1992; 158(5): 1081-1085, doi: 10.2214/ajr.158.5.1566671, indexed in Pubmed: 1566671 . 